\title{
Daniel von Superville - Karl Kaspar von Siebold - Franz Xaver von Haeberl - Philipp Franz von Walther.
}

Zu Ehren des in der Hauptstadt Bayerns tagenden Kongresses für innere Medizin. dem diese ganze Nummer gewidmet ist. bringt unsere heutige - siebente - Kunstbeilage die Porträts von vier Mannern. die namentlich in der Geschichte der drei medizinischen Fakultäten Bayerns eine hervorragende Rolle gespielt haben, die aber auch für die Entwicklung der deutschen Medizin uberhaupt von großer Bedeutung gewesen sind.

Daniel von Superville, Leibarzt des Markgrafen Friedrich und der Markgräfin Friederike Sophie Wilhelmine von Bayreuth, der Schwester Friedrichs des Großen. ist der Gründer der Universität in Erlangen. Seiue wichtigsten biographischen Daten zitieren wir wörtlich aus „G. W. A. Fikenscher, Geschichte der Königlich Preußischen Friedrich-Alexanders-Universität zu Erlangren von ihrem Crsprung bis auf gegenwartige Zeiten entworfen. Coburg 1795." s. $440 \mathrm{ff}$. "Daniel von Giperville, der $2\left(\mathfrak{r}_{3}\right.$ nengelahrtheit Doctor,

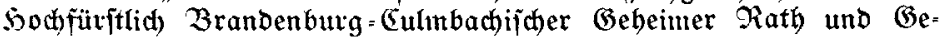
janbter beş Füritenthuns Banreuth zu joag in Sollano, wie auch Mitglied ber faiferlid) Leopoldinifchen Mlcabemie Der Paturforidher uno Der söniglich) Preufifichen Eocietät ber 2 iffenichaften zu Berlin,

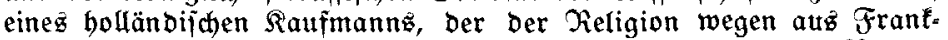
reid) entflohen war, Jacob von Guperville, Gohn, melcher 3 Kotter. Dam am 2. December 1696. geboren, und bajelbit in feiner Sugend in

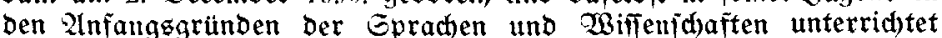
worben war. Diejer beiudte in reiferm Llter aubere Golländijhe Ed)ulen mo bilbete fich umter ben berübmten $\mathfrak{M}$ ännern ber bamaligen

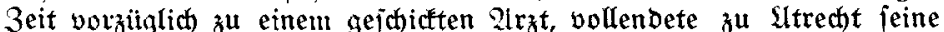

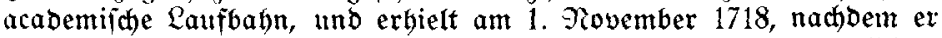
jeine Inauguraldifputation: de sanguine et sanguificatione vertbeibiget

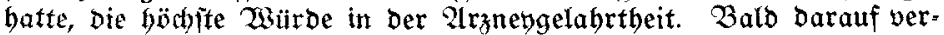

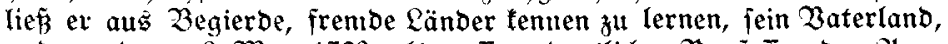

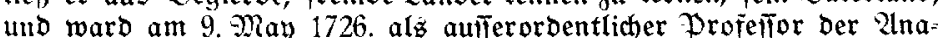
tomie und Chirurgie, und als Päbagog aun Carolinif̧en Gymnafium zu Etettin eingefuibrt, und gelangte algdann zu Der $\mathfrak{B}$ birbe eines $50 f=$

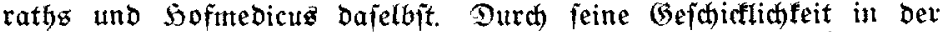
Seilfunbe nidht nur, jonbern aud) burch feine Senntnifie in Eprad)en und $\mathfrak{B}$ iffenidhaften, weldhe man immer mehr fennen lernte, gejdah es

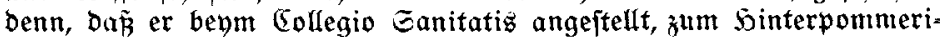
ja)en Qandphyficus, Dedicus bey Der franzöfifhen Golonie, und Gtadt=

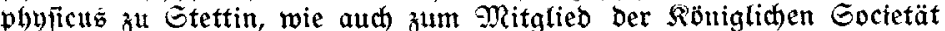
ber ZGiffenifhaften łu Berlin ernaunt wurbe. Der vielen (Befd)äfte aber, Die ihm Diefe Pemter veruriadfen, ungeachtet, hörte er nicht auf zu ftubieren, ja, er jorgte babey auch jogar für bie gelebrte $23 e l t$, und liể eine $\mathfrak{Z l b h a n d l u n g}$ von Quaffalbern brudfen, woburch die faifer. (id)e Zlcabemie ber Yaturforicher vorz üglich berogen wurbe, ifn audh zu ihrem Mitglieb anzunehmen. Shus welden Slrjachen er nun Poun=

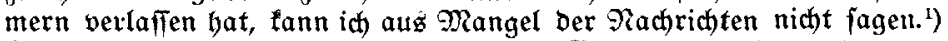
Genug, er fam $\mathrm{tm}$ bas $3 a h r ~ 1739$. nał Banreuth, und rourde bom Marfgraf Friederid) fum gebeimen Rath, Leibarzt und Director Der Bergwerte im $\mathcal{O b e r}=$ und slnterlande angenommen, woz" er 1740 . aud) Daś Directorium über bas Gbriftian (Ernejtinifche Gymna|ium erbielt.

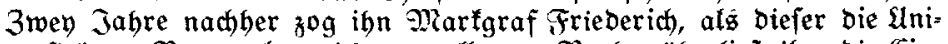

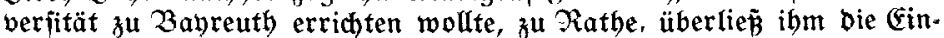
richtuttg Derielben, und beftellte ibn zum Director. $21 \mathrm{~m} 27$. Geptember

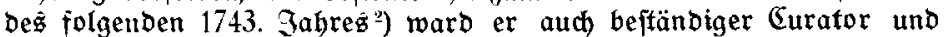
Ganzler Derielben, und weibte biefelbe ein." Da die Lniversität in Bayreuth nicht florierte, so wurde sie 1743 nach Erlangen verlegt und dort am 4 . November feierlich eroffnet. Superville hielt dabei als Kanzler eine lateinische Rede, „die sich durch Formengewandtheit und Gedankenfulle auszeichnete“. Die Lniversität wurde durch den

1) Er wurde der Markgräfin 1738 von ihrem Bruder, dem damaligen Kronprinz, in ihrer schweren Krankheit als Arzt empfohlen und ging dann. nachdem er die Patientin geheilt hatte, $1739 \mathrm{ganz}$ in markgräfliche Dienste über. K..Sehling, Daniel von Superville. Leipzig 1893.

2) Nach Sehling am 21. März 1742. 
energischen Mann sehr gefördert. Trotzdem kam es infolge seines diktatorischen Vorgehens schlie日lich zu seinem Sturz. 1748 verlieB er Stadt und Land, siedelte 1749 nach Braunschweig über und trat 1750 in die Dienste des Herzogs Carl von Braunschweig. 1776 starb er.

Karl Kaspar von Siebold, geboren den 4. November 1736 in Nideck (Herzogtum Jülich), gestorben den 5. April 1807 in Würzburg, ist der Stammvater der berühmten Würzburger Gelehrtenfamilie, der namentlich mehrere hochangesehene Aerzte entsproßt sind. Karl Kaspar selbst war ebenfalls der Sohn eines Arztes; bei seinem Vater genoß er auch den ersten Unterricht in der praktischen Chirurgie. Nachdem er mehrere Jahre sich in französischen Militärspitälern fortgebildet hatte, trat er 1760 als erster "Gehilfe" auf der chirurgischen Abteilung des Juliusspitals in Würzburg ein, hörte aber neben seiner praktischen Tatigkeit theoretische Vorlesungen über Naturwissenschaften und Medizin an der Universität. 1763 bis 1766 verrollkommnete er sein Wissen und Können durch Reisen in Frankreich, England und Holland. 1769 erlangte er den Doktortitel und wurde dann als Lehrer der Anatomie, Chirurgie und Geburtshilfe an der Würzburger Universitat angestellt. In allen drei Lehrfächern hat er durch seine wissenschaftlichen Tntersuchungen die Medizin bereichert. Besonders ragt er als Chirurg hervor (so hat er in Deutschland als erster die Symphysiotomie gemacht). Durch seine ausgezeichnete Lehrfähigkeit zog er eine große Zahl von Studenten nach Würzburg und trug so zur Hebung der Universität, insbesondere ihrer medizinischen Fakultät, wesentlich bej. Infolge seiner Bedeutung als Lehrer und Operateur erhielt er 1787 einen Ruf als Professor der Chirurgie und Oberwundarzt an die Charité in Berlin, lehnte ihn aber ab. An äuBeren Ehrungen fehlte es ihm auch in seinem dortigen Wirkungskreise nicht. 1777 wurde er Hofrat und Leibarzt des Fürsten Georg Carl, 1801 wurde er in den Reichsadel erhoben, 1803 zum Medizinalrat des Fürstentums Würzburg ernannt. - Seine Söhne sind Johann Georg Christoph $(1767-1798)$, a. o. Professor für allgemeine Therapie und Diätetik in Würzburg, Johann 'The od or Damian, Ober-Med.-Rat in Darmstadt (gestorben 1828), Johann Bartholomäus (1774-1814), Professor der Chirurgie und Oberwundarzt in Würzburg, und Adam Elias (1775-1828), der bedeutendste der Söhne, hervorragender Geburtshelfer, erst als Professor in Würzburg, dann (1816) in Berlin, wo er die geburtshilfliche Klinik gründete. - Auch die zweite Generation der Sieboldschen Familie hat einige ausgezeichnete Aerzte hervorgebracht.

Franz Xaver von Haeberl, geboren am 26. März 1759 in Eulkam, einem kleinen Dorf in Oberbayern, studierte in Ingolstadt Medizin, wurde dort, nach vorübergehendem Aufenthalt in Wien, 1784 zum Doktor promoviert und siedelte dann nach München iuber, wo er sich als praktischer Arzt niederließ. Durch seine Tatigkeit in zwei geistlichen Spitälern wurde er anf Beobachtungen und Studien über Krankenhaushygiene hingelenkt, als deren Ergebnisse zwei Arbeiten (1794 und 1799) erschienen, in denen die Mängel der bisherigen Einrichtungen und Vorschläge zul ihrer Reform eingehend dargelegt wurden. Die zweite der genannten Schriften "Wünsche und Vorschläge zur Errichtung eines allgemeinen Krankenhauses in München" wurde zum Gegenstand sorgfältiger Prüfung bei den Staats- und städtischen Behörden, mit dem Resultat, daß nach den Vorschlägen v. Haeberls das Allgemeine Krankenhaus in München errichtet wurde. Im Jahre 1813 war der Bau am Sendlinger Tor, mit 54 Krankensälen zu zwölf Betten, 36 Sonderzimmern und einem Operationssaal, vollendet, und Fr. v. Haeberl wurde zum ersten Direktor des neuen Krankenhauses ernannt. Neben diesem Amte hielt er auch als Lehrer der praktischen Arzneikunde vielbesuchte Vorlesungen. 1824 schied v. Haeberl aus München und zog sich auf sein Landgut bei Bayerdießen am Ammersee zurück; hier starb er, $87 \mathrm{Jahre}$ alt, am 23 April 1846. In dankbarer Anerkennung seiner Verdienste ist von der Stadt Muinchen in der Vorhalle des von ihm geschaffenen Krankenhauses eine Gedenktafel mit seinem Medaillonporträt gestiftet worden.

Philipp Franz von Walther, geboren den 3. Januar $1782 \mathrm{zu}$ Burweiler (Rheinpfalz), gestorben den 29. Dezember 1849, ist wohl die hervorragendste Erscheinung der Münchener medizinischen $\mathrm{Fa}$ kultät in der ersten Hälfte des vorigen Jahrhunderts. Mit 15 Jahren an der Heidelberger Universität immatrikuliert, studierte er dort, später in Wien Medizin und wurde 1803 an der damaligen Universität in Landshut zum Doktor promoviert. Noch in demselben Jahre wurde er, 21 Jahre alt, zum o. Prof., Med.-Rat und Oberwundarzt am Spital in Bamberg ernannt. 1804 wurde er Professor der Physiologie und Chirurgie in Landshut und entfaltete in letzterer Eigenschaft, namentlich auch bei Augenkranken, eine ausgezeichnete, erfolgreiche Tätigkeit. 1811 wurde er zum Rector magnificus gewählt. Nachdem er einen Ruf nach Halle und Heidelberg abgelehnt hatte, ging er 1818 nach Bonn, wo er sich nach dem Urteil seiner
Zeitgenossen zu einem glänzenden Meister der Chirargie und Augenheilkunde entwickelte. Aus dieser Periode seines Lebens stammen auch seine zahlreichsten wissenschaftlichen Mitteilungen; sie wurden in dem von v. Walther und $v$. Graefe seit dem Jahre 1820 herausgegebenen „Journal für Chirurgie und Augenheilkunde" veröffentlicht. 1830 folgte er einem Rufe König Ludwig I. an die - von Landshut dorthin verlegte - Universität in München, zugleich mit der Funktion eines Königlichen Leibarztes und mit dem Titel eines Geheimen Rats. Auch hier wirkte er als Kliniker mit hervorragendem Erfolge. 1836 legte er seine Stellung als Direktor der chirurgischen und Augenklinik ${ }^{1}$ ) nieder, behielt aber seine theoretischen Vorlesungen bis zum Tode bei. Walthers wissenschaftliche Tatigkeit war, neben der praktischen, äußerst fruchtbringend. Die Medizin hat von ihm eine wesentliche Förderung erfahren, insbesondere die Chirurgie, für deren innere und äußere Entwicklung er unablässig wirkte. Auf ihn ist die Aufhebung der chirurgischen Schulen in Bayern, aus denen die niedere Zunft der Wundchirurgen und Bader hervorging, zurückzuführen. Auch sonst war er bemïht, das Niveau des ärztlichen Standes zu heben und seine Stellung zu verbessern. In diesem Sinne trat el 1848 auf dem ReformkongreB der bayerischen Aerzte in München für die Freigebung der ärztlichen Praxis ein. Ueber seine Spezialwissenschaft hinaus erstreckten sich Walthers allgemeine naturwissenschaftliche und philosophische, durch seinen Freund Schelling beeinflußte Studien und Arbeiten. Die Zahl seiner Abhandlungen auf dem Gebiete der Anatomie, Physiologie, Chirurgie, A ugenheilkunde, Inneren Medizin etc. ist enorm. In der Festrede, die v. Nussbaum gelegentlich der vom Münchenes ärztlichen Verein am 4. Januar 1882 veranstalteten Feier des $100 \mathrm{jah}$ rigen Geburtstages v. Walthers hielt, sagt er von ihm: „Als Arzt am Krankenbett war er unerreichbar, für die Wissenscliaft aber kann man ihn einell segenbringenden Reformator nennen". J. S.
1) Die beiden Lehrfächer wurden in München erst 1871 voncinander getrennt. 